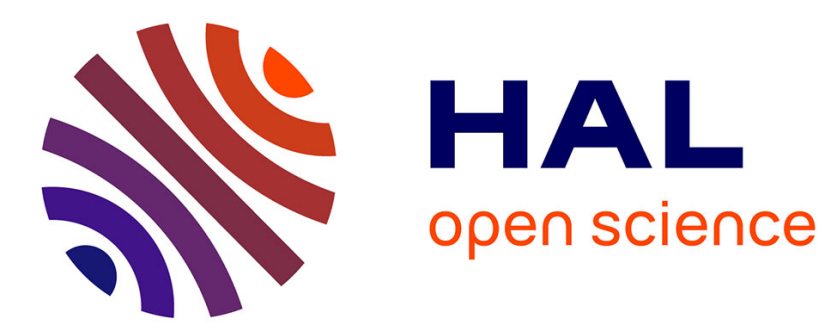

\title{
Last minute panic in zero sum games
}

Stefan Ankirchner, Christophette Blanchet-Scalliet, Kai Kümmel

\section{To cite this version:}

Stefan Ankirchner, Christophette Blanchet-Scalliet, Kai Kümmel. Last minute panic in zero sum games. ESAIM: Control, Optimisation and Calculus of Variations, 2019, 25, 10.1051/cocv/2018015 . hal-01421056

\section{HAL Id: hal-01421056 \\ https://hal.science/hal-01421056}

Submitted on 21 Dec 2016

HAL is a multi-disciplinary open access archive for the deposit and dissemination of scientific research documents, whether they are published or not. The documents may come from teaching and research institutions in France or abroad, or from public or private research centers.
L'archive ouverte pluridisciplinaire HAL, est destinée au dépôt et à la diffusion de documents scientifiques de niveau recherche, publiés ou non, émanant des établissements d'enseignement et de recherche français ou étrangers, des laboratoires publics ou privés. 


\title{
Last minute panic in zero sum games
}

\author{
Stefan Ankirchner * Christophette Blanchet-Scalliet ${ }^{\dagger}$ \\ Kai Kümmel ${ }^{\ddagger}$
}

December 29, 2017

We set up a game theoretical model to analyze the optimal attacking intensity of sports teams during a game. We suppose that two teams can dynamically choose among more or less offensive actions and that the scoring probability of each team depends on both teams' actions. We assume a zero sum setting and characterize a Nash equilibrium in terms of the unique solution of an Isaacs equation. We present results from numerical experiments showing that a change in the score has a strong impact on strategies, but not necessarily on scoring intensities. We give examples where strategies strongly depend on the score, the scoring intensities not at all.

\section{Introduction}

Towards the end of professional sports games one can frequently observe that teams change their proneness for risk: the team being behind starts playing more offensively, and the team ahead starts playing more defensively. E.g. if a soccer team is behind by one goal, then close to the end of the game some of the defenders move to the front and act as forwards. Conversely, the soccer team that is ahead tends to pull back all players and to reinforce the defense unit. Similarly, near the end of ice hockey games the loosing team usually is pulling the goalie, i.e. is removing the goaltender for an extra attacker.

The loosing team often switches to a more offensive strategy even if this increases the probability of conceding a goal by much more than the probability of scoring a goal. Such a strategy change seems reasonable, since close to the end of the game the team

*Stefan Ankirchner, Institute for Mathematics, University of Jena, Ernst-Abbe-Platz 2, 07745 Jena, Germany. Email: s.ankirchner@uni-jena.de, Phone: +49 (0)3641 946275.

${ }^{\dagger}$ Christophette Blanchet-Scalliet, Université de Lyon - CNRS, UMR 5208, Institut Camille Jordan - Ecole Centrale de Lyon, 36 avenue Guy de Collongue, 69134 Ecully Cedex, FRANCE. Email: christophette.blanchet@ec-lyon.fr, Phone: +33472186405

${ }^{\ddagger}$ Kai Kümmel, Institute for Mathematics, University of Jena, Ernst-Abbe-Platz 2, 07745 Jena, Germany. Email: kai.kuemmel@uni-jena.de, Phone: +49 (0)3641 946188 
has nothing to loose anymore, while it can gain a lot by equalizing. There is a debate, however, on when it is optimal for the team behind to start playing more offensively. Some trainers are reluctant to switch to more offensive strategies, whereas others are willing to take risks early.

The aim of the present article is to provide a method for determining the optimal degree of aggressiveness during a game. We strive for this aim by taking into account that teams can react to the opponent's strategy changes. E.g. if one team starts playing more offensively, then the other team can respond by playing more defensively. Moreover, we allow for smooth transitions between defensive and offensive strategies. The reason is that in many sports, e.g. soccer, teams can smoothly modify the aggressiveness by gradually changing the positions of the players.

We set up a game theoretical model (see Section 1), where sports teams can make their strategies dependent on the score and the remaining time. We model a game, where two teams can choose among a continuum of actions. The actions determine the intensity of two stochastic processes counting the goals of each team. For simplicity we restrict our analysis to zero sum games, i.e. games where the sum of the points assigned to both teams is constant. A paradigm are playoff elimination games, bringing both teams into 'do-or-die' situations.

The zero-sum assumption means that for each team the aim of maximizing its own expected payoff is equivalent to the aim of minimizing the opponent's expected payoff. Therefore, in this case a Nash equilibrium can be determined by solving a minimax problem. We show in Section 2 that, under some general assumptions, the Isaacs equation associated to the minimax problem has a unique classical solution. A verification argument then allows to identify the solution with the value function of the minimax problem. Moreover, a unique pure strategy Nash equilibrium is characterized as the value function's saddle point.

In general, the solution of the Isaacs equation is not given in closed form and hence needs to be approximated numerically. In Section 3 we discuss several numerical methods for approximating the solution. We provide sufficient conditions for finite difference schemes and an iterative scheme to converge.

In Section 4 we present results from numerical experiments. Here is a summary of our main findings:

- As soon as a team falls behind, it is optimal for them to switch to a more offensive strategy - and this even if the strategy change makes the opponent relatively stronger (i.e. increases the opponent's scoring likelihood by more than the own scoring likelihood). Similarly, as soon as a team gains the lead, it is optimal for them to play more defensively.

- The closer the end of the game, the bigger the impact of the score on the teams' strategies. Close to the end, the losing team should play extremely offensively, whereas the winning team extremely defensively.

- Although a change of the score has a strong impact on strategies, the impact on the scoring intensities is less pronounced. Indeed, there are situations where the 
teams' actions change considerably after a goal, but the changes mutually cancel out the impact on the scoring intensities.

- The lower the overall scoring intensities, the more likely a weaker team can beat a stronger one.

There are already contributions in the literature proving that it can be indeed optimal for the loosing team to play more offensively towards the end of the game. A series of articles provide evidence that it is reasonable for a loosing ice hockey team to pull the goalie shortly before the end of the game, even if this increases the opponent's scoring intensity far more than the own intensity (see e.g. [12], [15] and [4]). Morrison and Wheat [12] assume that pulling the goalie is irreversible, which allows to determine the optimal pulling time via a simple one-dimensional optimization. Washburn [15] uses a dynamic programming approach for determining the optimal time for pulling the goalie. Beaudoin and Swartz [4] describe a simulation method for characterizing optimal pulling times. To the best of our knowledge there are no game theoretical models aiming at analyzing the optimal attacking intensity of sports teams.

In case both teams are equally strong, the loosing team tends to increase the overall scoring intensities, whereas the winning team tends to decrease them. Strategies that increase the fluctuations in unfavorable situations and reduce fluctuations in favorable ones have been shown to be optimal in some control problems without interaction. McNamara [11] considers a control problem, where the volatility of a Brownian martingale can be chosen to take any value between $\sigma_{1}$ and $\sigma_{2}$, with $0<\sigma_{1}<\sigma_{2}$. He characterizes the reward functions for which it is optimal to choose the minimal volatility when the martingale is above zero, and the maximal volatility when it is below zero. In [1] the authors consider an exponential martingale model in which a bang-bang diffusion control is optimal.

The numerical methods presented in Section 3 are similar to approximation methods that have been developped already for other types of differential games. The articles of Part I in [3] provide an overview on numerical approximation methods for deterministic differential games. See also [6] or [7] for an overview on numerical methods for pursuit-evasion games. The Isaacs equation associated to a zero-sum differential game with continuous state dynamics can be approximated with a Semi-Lagrangian scheme. Alternatively, if the value function can be identified with a fixed point of a contracting mapping, then one can construct an approximing sequence by iteratively applying the mapping. In Section 3 we apply both approximation ideas to the Isaacs equation of the stochastic game introduced in the present article. In constrast to the models considered in [3] and [6, 7], in our model the state process is a two-dimensional Poisson process taking values in $\mathbb{Z}^{2}$, and hence no discretization of the state space is needed.

\section{The model}

We model the score of a sports game with two point processes counting the goals/points scored by the own and the opposing team, respectively. The intensity of the point 
processes will depend on the strategies chosen by both teams. We suppose that at any time between 0 (the beginning of the game) and $T \in \mathbb{R}_{>0}$ (the end) both teams can choose an action determining the strategy. We model the set of actions of the own team and opposing team as bounded intervals $A$ and $B$ in $\mathbb{R}$ respectively.

The family of control functions of the own team is denoted by $\mathcal{A}$ and consists of all functions $\alpha:[0, T] \times \mathbb{Z} \rightarrow A$ such that for each $k \in \mathbb{Z}$ the path $t \mapsto \alpha(t, k)$ is càdlàg. Similarly, a control of the opposing team is a càdlàg function $\beta:[0, T] \times \mathbb{Z} \rightarrow B$, and we denote the set of all opposing team controls by $\mathcal{B}$. For $i=1,2$ let $\lambda_{i}: A \times B \rightarrow \mathbb{R}_{\geq 0}$ be measurable mappings. We interpret $\lambda_{1}(a, b)$ as the intensity with which the own team scores a goal, given that both teams choose the actions $a$ and $b$ respectively. Similarly, $\lambda_{2}(a, b)$ stands for the opposing team's goal scoring intensity. In order to apply a minimax theorem we make the following assumption:

Hypothesis $(\Lambda)$. For each fixed $b \in B$ the mappings

$$
a \mapsto \lambda_{1}(a, b) \text { and } a \mapsto-\lambda_{2}(a, b) \text { are quasi-concave and upper semi-continuous }
$$

and for each fixed $a \in A$ the mappings

$$
b \mapsto \lambda_{1}(a, b) \text { and } b \mapsto-\lambda_{2}(a, b) \text { are quasi-convex and lower-semicontinuous. }
$$

Notice that Hypothesis $(\Lambda)$ is satisfied if $\lambda_{1}$ and $-\lambda_{2}$ are concave in $a$ and convex in $b$. This special case means that the marginal scoring benefit from playing more offensively is decreasing. The marginal benefit to the opponent, however, is increasing.

We model the goal counting processes as point processes with stochastic intensity. We recall the precise definition of such processes before proceeding with the model description. Throughout we are assuming that $(\Omega, \mathcal{F}, P)$ is a probability space rich enough for carrying the probabilistic objects considered.

Definition 1.1 (cf. with [5]). Let $\left(N_{t}\right)_{t \in[0, T]}$ be an non-decreasing, right-continuous process with values in the set of non-negative integers such that $N_{0}=0$ and $\Delta N_{t}=$ $N_{t}-N_{t-} \in\{0,1\}$ for all $t \in(0, T]$. Let $\left(\mathcal{F}_{t}\right)_{t \in[0, T]}$ be a filtration such that $N$ is adapted to $\left(\mathcal{F}_{t}\right)$. Let $\left(\lambda_{t}\right)_{t \in[0, T]}$ be a $\left(\mathcal{F}_{t}\right)$-progressively measurable, non-negative process satisfying

$$
\int_{0}^{t} \lambda_{s} \mathrm{~d} s<\infty, \quad \mathrm{P}-a . s .
$$

for each $t \in[0, T]$. The process $\left(N_{t}\right)$ is said to be a point process with $\left(\mathcal{F}_{t}\right)$-intensity $\lambda$ if

$$
N_{t}-\int_{0}^{t} \lambda_{s} d s \text { is a local martingale on }[0, T] .
$$

Notice that $(2)$ is equivalent to requiring that for all non-negative, $\left(\mathcal{F}_{t}\right)$-predictable processes $\left(\varphi_{t}\right)_{t \in[0, T]}$ we have

$$
\mathbb{E}\left[\int_{0}^{T} \varphi_{s} \mathrm{~d} N_{s}\right]=\mathbb{E}\left[\int_{0}^{T} \varphi_{s} \lambda_{s} \mathrm{~d} s\right] .
$$


We now proceed by describing the model. We assume that there exists a single filtration $\left(\mathcal{F}_{t}\right)_{t \in[0, T]}$ such that for every $\alpha \in \mathcal{A}$ and $\beta \in \mathcal{B}$ there exist point processes $N_{t}^{\lambda_{1}(\alpha, \beta)}$ and $M_{t}^{\lambda_{2}(\alpha, \beta)}$ with $\left(\mathcal{F}_{t}\right)$-intensity $\lambda_{1}\left(\alpha\left(t, K_{t}^{\alpha, \beta}\right), \beta\left(t, K_{t}^{\alpha, \beta}\right)\right)$ and $\lambda_{2}\left(\alpha\left(t, K_{t}^{\alpha, \beta}\right), \beta\left(t, K_{t}^{\alpha, \beta}\right)\right)$ respectively, where

$$
K_{t}^{\alpha, \beta}=N_{t}^{\lambda_{1}(\alpha, \beta)}-M_{t}^{\lambda_{2}(\alpha, \beta)}
$$

denotes the score difference at time $t$. For an explicit construction of the filtration and the processes we refer to the appendix.

We model the reward with a bounded and non-decreasing function $R: \mathbb{Z} \rightarrow \mathbb{R}$. We assume that there exists a constant $c$ such that

$$
R(-k)=c-R(k) \quad \text { for all } k \in \mathbb{Z} .
$$

Such reward functions describe the situation of zero sum games, when the total amount of points assigned at the end of the game is constant. A particular choice representing elimination games is

$$
R(k):= \begin{cases}2, & \text { if } k \geq 1 \\ 1, & \text { if } k=0 \\ 0, & \text { if } k \leq-1\end{cases}
$$

If $R(k)=2$, then the own team enters the next round and if $R(k)=0$, then it is eliminated. $R(k)=1$ means that the game has to be extended until a winner has been determined.

For every $(t, k) \in[0, T] \times \mathbb{Z}$ and control pair $(\alpha, \beta) \in \mathcal{A} \times \mathcal{B}$ the expected gain of the own team is given by

$$
J(t, k, \alpha, \beta)=E\left[R\left(K_{T}^{t, k, \alpha, \beta}\right)\right],
$$

and the expected gain of the opposing team by

$$
I(t, k, \alpha, \beta)=E\left[R\left(-K_{T}^{t, k, \alpha, \beta}\right)\right],
$$

where $K_{T}^{\alpha, \beta, k, t}:=k+N_{T}^{\lambda_{1}(\alpha, \beta)}-N_{t}^{\lambda_{1}(\alpha, \beta)}-M_{T}^{\lambda_{2}(\alpha, \beta)}+M_{t}^{\lambda_{2}(\alpha, \beta)}$.

The zero sum assumption (4) implies that $I(t, k, \alpha, \beta)=c-J(t, k, \alpha, \beta)$ and hence maximizing $I$ is equivalent to minimizing $J$.

In the next section we show, under Hypothesis $(\Lambda)$, that there exists a pair $\left(\alpha^{*}, \beta^{*}\right) \in$ $\mathcal{A} \times \mathcal{B}$ that is a saddle point for $J$, i.e. it satisfies

$$
J\left(t, k, \alpha^{*}, \beta^{*}\right)=\min _{\beta \in \mathcal{B}} J\left(t, k, \alpha^{*}, \beta\right)=\max _{\alpha \in \mathcal{A}} J\left(t, k, \alpha, \beta^{*}\right) .
$$

In particular, this implies that $\left(\alpha^{*}, \beta^{*}\right)$ is a pure strategy Nash equilibrium. 
For the proof we introduce the minimax and maximin value function for the own team, namely

$$
\begin{aligned}
V_{+}(t, k) & =\inf _{\beta \in \mathcal{B}} \sup _{\alpha \in \mathcal{A}} J(t, k, \alpha, \beta) \quad \text { and } \\
V_{-}(t, k) & =\sup _{\alpha \in \mathcal{A}} \inf _{\beta \in \mathcal{B}} J(t, k, \alpha, \beta) .
\end{aligned}
$$

Notice that both satisfy the terminal condition

$$
V_{ \pm}(T, k)=R(k)
$$

In the next section we show that $V_{+}=V_{-}$and that there exists a saddle point. We refer to the function $V=V_{+}=V_{-}$as the value of the game.

\section{The value function is a solution of the Isaacs equations}

For every $(t, k, a, b) \in[0, T] \times \mathbb{Z} \times A \times B$ and function $v:[0, T] \times \mathbb{Z} \rightarrow \mathbb{R}$ we define

$$
G(t, k, a, b, v)=\left\{\lambda_{1}(a, b)(v(t, k+1)-v(t, k))+\lambda_{2}(a, b)(v(t, k-1)-v(t, k))\right\} .
$$

Recall that the Isaacs equation associated to the minimax value function $V_{+}$is given by

$$
-w_{t}(t, k)=\min _{b \in B} \max _{a \in A} G(t, k, a, b, w),
$$

for $(t, k) \in[0, T] \times \mathbb{Z}$. A natural choice of the solution space for the Isaacs equation is $\mathcal{C}^{1}\left([0, T], l_{\infty}\right)$, the space of continuously differentiable functions on $[0, T]$ taking values in $l_{\infty}$, the space of bounded integer-indexed sequences.

By interchanging the min and max in (8) we obtain the Isaacs equation for the maximin value function $V_{-}$. We show in this section, by using Banach's fixed point theorem, that both Isaacs equations have a unique solution satisfying the terminal condition (6). Hypothesis $(\Lambda)$ guarantees that a minimax theorem applies and both solutions coincide. By using a verification we can thus identify the solution with both value functions $V_{+}$ and $V_{-}$. The following theorem summarizes the main results.

Theorem 2.1. There exists a unique solution $w \in \mathcal{C}^{1}\left([0, T], l_{\infty}\right)$ of $(8)$ with terminal condition $R$, and $w=V_{+}=V_{-}$. Moreover, a saddle point (and hence Nash equilibrium) is given by

$$
\begin{aligned}
& \beta^{*}(t, k) \in \operatorname{argmin}_{b \in B} G(t, k, \hat{\alpha}(t, k, b), w) \\
& \alpha^{*}(t, k)=\hat{\alpha}\left(t, k, \beta^{*}(t, k)\right),
\end{aligned}
$$

where $\hat{\alpha}(t, k, b) \in \operatorname{argmax}_{a \in A} G(t, k, a, b, w)$. 
We first show that Equation (8) has a solution. To this end let $\mathcal{X}:=\mathcal{C}_{b}\left([0, T], l_{\infty}\right)$ be the space of continuous and bounded functions on $[0, T]$ with values in $l_{\infty}$. We apply Banach's fixed point theorem on the space $\mathcal{X}$ with the mapping

$$
\Phi: \mathcal{X} \rightarrow \mathcal{X} ; \quad v \mapsto\left((t, k) \mapsto R(k)-\int_{t}^{T} H(s, k, v) \mathrm{d} s\right)
$$

where $H:[0, T] \times \mathbb{Z} \times \mathcal{X} \rightarrow \mathbb{R}$ is given by

$$
H(t, k, v):=\min _{b \in B} \max _{a \in A} G(t, k, a, b, v) .
$$

Lemma 2.2. For each fixed $(t, k) \in[0, T] \times \mathbb{Z}$ the mapping $v \mapsto H(t, k, v)$ is Lipschitz continuous with Lipschitz constant $L:=2 \max \left\{\lambda_{1}(a, b)+\lambda_{2}(a, b): a \in A, b \in B\right\}$.

Proof. Let $v_{1}, v_{2} \in \mathcal{X}$. We can assume $H\left(t, k, v_{1}\right)>H\left(t, k, v_{2}\right)$ (else interchange $v_{1}$ and $\left.v_{2}\right)$. By definition of $H$ we obtain

$$
\begin{aligned}
& \left|H\left(t, k, v_{1}\right)-H\left(t, k, v_{2}\right)\right|=H\left(t, k, v_{1}\right)-H\left(t, k, v_{2}\right) \\
& =\min _{b_{1} \in B} \max _{a_{1} \in A}\left\{\lambda_{1}\left(a_{1}, b_{1}\right)\left(v_{1}(t, k+1)-v_{1}(t, k)\right)+\lambda_{2}\left(a_{1}, b_{1}\right)\left(v_{1}(t, k-1)-v_{1}(t, k)\right)\right\} \\
& \quad-\min _{b_{2} \in B} \max _{a_{2} \in A}\left\{\lambda_{1}\left(a_{2}, b_{2}\right)\left(v_{2}(t, k+1)-v_{2}(t, k)\right)+\lambda_{2}\left(a_{2}, b_{2}\right)\left(v_{2}(t, k-1)-v_{2}(t, k)\right)\right\} \\
& \leq \max _{b_{2} \in B}\left\{\max _{a_{1} \in A}\left\{\lambda_{1}\left(a_{1}, b_{2}\right)\left(v_{1}(t, k+1)-v_{1}(t, k)\right)+\lambda_{2}\left(a_{1}, b_{2}\right)\left(v_{1}(t, k-1)-v_{1}(t, k)\right)\right\}\right. \\
& \left.\quad-\max _{a_{2} \in A}\left\{\lambda_{1}\left(a_{2}, b_{2}\right)\left(v_{2}(t, k+1)-v_{2}(t, k)\right)+\lambda_{2}\left(a_{2}, b_{2}\right)\left(v_{2}(t, k-1)-v_{2}(t, k)\right)\right\}\right\} \\
& \leq 2 \max _{b \in B} \max _{a \in A}\left\{\lambda_{1}(a, b)+\lambda_{2}(a, b)\right\}\left\|v_{1}-v_{2}\right\|_{\infty} .
\end{aligned}
$$

Lemma 2.3. There exists a unique solution of Equation (8) with terminal condition $R$.

Proof. We separate the proof in two steps. First we define a suitable Banach space. Then we show that the mapping $\Phi$ is a contraction, which entails existence and uniqueness.

Step 1. The space $\mathcal{C}_{b}\left([0, T], l_{\infty}\right)$ endowed with the norm $\|\cdot\|_{\infty, L}$ given by

$$
\|v\|_{\infty, L}:=\sup _{k \in \mathbb{Z}} \sup _{t \in[0, T]}\left\{\mathrm{e}^{-2 \mathrm{~L}(T-t)}|v(t, k)|\right\}
$$

is a Banach space.

Indeed, both $l_{\infty}$ and $\mathcal{C}_{b}=\mathcal{C}_{b}([0, T], \mathbb{R})$ endowed with the standard norms are Banach spaces. We can ignore the bounded weight. Now let $\left(v^{n}\right)_{n \in \mathbb{N}}$ be a Cauchy sequence in $\mathcal{X}$, i.e. for each $\varepsilon>0$ there is a $N(\varepsilon)$ such that

$$
\sup _{k \in \mathbb{Z}} \sup _{t \in[0, T]}\left|v^{m}(t, k)-v^{n}(t, k)\right|<\varepsilon
$$


for each $n, m>N(\varepsilon)$. In particular, $\left\|v^{m}(\cdot, k)-v^{n}(\cdot, k)\right\|_{\infty}<\varepsilon$ for each $k \in \mathbb{Z}$ and $n, m>N(\varepsilon)$. Since $\mathcal{C}_{b}$ is a Banach space, there is a $v(\cdot, k)$ for each $k \in \mathbb{Z}$, such that $v^{n}(\cdot, k) \rightarrow v(\cdot, k)$ in $\mathcal{C}_{b}$ uniformly in $k$ (since $N(\varepsilon)$ does not depend on $\mathrm{k}$ ). Since $v^{n}(\cdot, k) \rightarrow v(\cdot, k)$ in $\mathcal{C}_{b}$ uniformly, we obtain

$$
\sup _{k \in \mathbb{Z}} \sup _{t \in[0, T]}\left|v^{n}(t, k)-v(t, k)\right|=\sup _{t \in[0, T]} \sup _{k \in \mathbb{Z}}\left|v^{n}(t, k)-v(t, k)\right| \rightarrow 0 \text { as } n \rightarrow \infty,
$$

and obviously, $v \in \mathcal{X}$ and the first part is done.

Step 2. Thanks to Lemma 2.2 we can conclude

$$
\begin{aligned}
\left\|\Phi\left(v_{1}\right)-\Phi\left(v_{2}\right)\right\|_{\infty, L} & \leq \sup _{k \in \mathbb{Z}} \sup _{t \in[0, T]} \mathrm{e}^{-2 L(T-t)} \int_{t}^{T}\left|H\left(s, k, v_{1}\right)-H\left(s, k, v_{2}\right)\right| \mathrm{d} s \\
& \leq\left\|v_{1}-v_{2}\right\|_{\infty, L} \sup _{t \in[0, T]} \mathrm{e}^{-2 L(T-t)} \int_{t}^{T} L \mathrm{e}^{2 L(T-s)} \mathrm{d} s \\
& =\left\|v_{1}-v_{2}\right\|_{\infty, L} \sup _{t \in[0, T]} \frac{1}{2}\left(1-\mathrm{e}^{-2 L(T-t)}\right) \leq \frac{1}{2}\left\|v_{1}-v_{2}\right\|_{\infty, L} .
\end{aligned}
$$

According to Banach's fixed point theorem, there is a unique fixed point $w \in \mathcal{C}_{b}\left([0, T], l_{\infty}\right)$ satisfying

$$
w=\Phi(w)
$$

i.e. $w$ satifies the Isaacs Equation (8) for each $(t, k) \in[0, T] \times \mathbb{Z}$ with terminal condition $V(T, k)=R(k)$ for each $k \in \mathbb{Z}$. Moreover, the fact that $w$ is a fixed point of $\Phi$ entails $w \in \mathcal{C}^{1}\left([0, T], l_{\infty}\right)$.

We now verify that $w$ coincides with $V_{+}$.

Lemma 2.4. The solution $w$ of (8) coincides with the value function, i.e. $w=V_{+}$.

Proof. Note that for any pair $(\alpha, \beta) \in \mathcal{A} \times \mathcal{B}$ we have, with Ito's formula,

$$
\begin{aligned}
w\left(T, K_{T}^{\alpha, \beta, t, k}\right)= & w\left(t, K_{t}^{\alpha, \beta, t, k}\right)+\int_{t}^{T} w_{t}\left(s, K^{\alpha, \beta, t, k}\right) \mathrm{d} s \\
+ & +\sum_{t<s \leq T}\left\{\left[w\left(s, K_{s-}^{\alpha, \beta, t, k}+1\right)-w\left(s, K_{s-}^{\alpha, \beta, t, k}\right)\right] \Delta N_{s}\right. \\
& \left.\quad+\left[w\left(s, K_{s-}^{\alpha, \beta, t, k}-1\right)-w\left(s, K_{s-}^{\alpha, \beta, t, k}\right)\right] \Delta M_{s}\right\} .
\end{aligned}
$$

Hence we obtain

$$
\mathbb{E}\left[R\left(K_{T}^{\alpha, \beta, t, k}\right)\right]=w(t, k)+\mathbb{E} \int_{t}^{T}\left(w_{t}\left(s, K_{s-}^{\alpha, \beta, t, k}\right)+G\left(s, K_{s-}^{\alpha, \beta, t, k}, \alpha, \beta, w\right)\right) \mathrm{d} s
$$


We now show that $w \leq V_{+}$. To this end fix $\beta \in \mathcal{B}$ and define

$$
\alpha_{\beta}^{*}(t, k) \in \underset{a \in A}{\operatorname{argmax}} G(t, k, a, \beta(t, k), w) .
$$

Since $A$ is compact and $t \rightarrow G(t, k, a, \beta(t, k), w)$ is cadlag, we can assume $\alpha^{*} \in \mathcal{A}$. Notice that $0 \leq w_{t}(t, k)+\max _{a \in A} G(t, k, a, \beta(t, k), w)=w_{t}(t, k)+G\left(t, k, \alpha_{\beta}^{*}(t, k), \beta(t, k), w\right)$. Together with Equation (13) this entails

$$
w(t, k) \leq \mathbb{E}\left(R\left(K_{T}^{\alpha_{\beta}^{*}, \beta, t, k}\right)\right) \leq \max _{\alpha \in \mathcal{A}} \mathbb{E}\left(R\left(K_{T}^{\alpha, \beta, t, k}\right) .\right)
$$

Since $\beta$ is arbitrary, this further implies $w(t, k) \leq V_{+}(t, k)$.

We proceed by showing that $w \geq V_{+}$. Fix $\alpha \in \mathcal{A}$ and define

$$
\beta_{\alpha}^{*}(t, k) \in \underset{b \in B}{\operatorname{argmin}} G(t, k, \alpha(t, k), b, w) .
$$

As for $\alpha^{*}$, we can assume that $\beta^{*} \in \mathcal{B}$. Observe that

$$
0 \geq w_{t}(t, k)+\min _{b \in B} G(t, k, \alpha(t, k), b, w)=w_{t}(t, k)+G\left(t, k, \alpha(t, k), \beta_{\alpha}^{*}(t, k), w\right),
$$

and hence, with Equation (13),

$$
w(t, k) \geq \mathbb{E}\left(R\left(K_{T}^{\alpha, \beta_{\alpha}^{*}, t, k}\right)\right) .
$$

Since $\alpha$ is arbitrarily chosen, we get

$$
w(t, k) \geq \max _{\alpha \in \mathcal{A}} \mathbb{E}\left(R\left(K_{T}^{\alpha, \beta_{\alpha}^{*}, t, k}\right)\right) \geq \min _{\beta \in \mathcal{B}} \max _{\alpha \in \mathcal{A}} \mathbb{E}\left(R\left(K_{T}^{\alpha, \beta, t, k}\right)\right)=V_{+}(t, k) .
$$

End of the proof of Theorem 2.1. So far we have shown that $w=V_{+}$. Similar arguments show that there exists a unique solution $u$ of the Equation (8) with min and max interchanged, and that $u=V_{-}$.

Observe that $V_{+}(t, k) \leq V_{+}(t, k+1)$ for each $t \in[0, T]$ and $k \in \mathbb{Z}$ (by the monotonicity assumption on $R$ and definition of $\left.V_{+}\right)$. Therefore, $w$ is non-decreasing in $k$. Hypothesis $(\Lambda)$ entails that we can apply Sion's minimax theorem (see [14] and Section 7.1.9 in [2]) to conclude that

$$
\max _{a \in A} \min _{b \in B} G(t, k, a, b, w)=\min _{b \in B} \max _{a \in A} G(t, k, a, b, w) .
$$

This further implies that $w=u$, and hence $V_{+}=V_{-}$.

The calculations in the proof of Lemma 2.4 show that the strategies (9) and (10) form a saddle point. 


\section{Numerical Approximation of the Isaacs equation}

In general, we do not have a closed form representation of the solution of the Isaacs Equation (8). In this section we discuss three numerical methods for approximating the solution of (8).

\subsection{Explicit finite difference scheme}

One can numerically approximate the solution of the Isaacs Equation (8) by using a finite difference scheme. Let $N \in \mathbb{N}, \Delta t=\frac{T}{N}$ and $t_{j}=j \Delta t$ for $j \in\{0, \ldots, N\}$.

As the Equation (8) is defined on $\mathbb{Z}$, we need to introduce some artificial boundaries. For $\mathrm{C} \in \mathbb{N}$ let $\tilde{V}^{\mathrm{C}}$ be the solution of the following scheme with Dirichlet boundary conditions:

$$
\begin{cases}\widetilde{V}^{\mathrm{C}}\left(t_{n-1}, k\right)=\widetilde{V}^{\mathrm{C}}\left(t_{n}, k\right)+\Delta t H\left(t_{n}, k, \widetilde{V}^{\mathrm{C}}\right), & \text { for } 0 \leq n \leq N-1, k=-\mathrm{C}+1, \ldots, \mathrm{C}-1 \\ \widetilde{V}^{\mathrm{C}}\left(t_{n}, \mathrm{C}\right)=R(\mathrm{C}), \widetilde{V}^{\mathrm{C}}\left(t_{n},-\mathrm{C}\right)=R(-\mathrm{C}), & \text { for } 0 \leq n \leq N-1, \\ \widetilde{V}^{\mathrm{C}}(T, k)=R(k), & \text { for } k=-\mathrm{C}, \ldots, \mathrm{C} .\end{cases}
$$

We next show that $\tilde{V}^{\mathrm{C}}$ converges to the value function as $N$ and $\mathrm{C} \rightarrow \infty$. The proof is divided into two step. First, we introduce Dirichlet boundary conditions in the Isaacs equation which allows to reduce the state space to a finite space (see Subsection 3.1.1). In a second step we prove convergence of the time discretization (see Subsection 3.1.2).

\subsubsection{Localization}

To reduce the state space, we choose homogeneous Dirichlet boundary conditions. These conditions are convenient as they still allow for a probabilistic interpretation of the Isaacs equation. Indeed, to prove convergence as $\mathrm{C} \rightarrow \infty$, we use a method introduced in Jaillet et al. [10] and Zhang [17] for American options.

We define $\tau^{\mathrm{C}}=\inf \left\{s \in[t, T],\left|K_{s}^{\alpha, \beta}\right| \geq \mathrm{C}\right\}$ and

$$
\bar{V}^{\mathrm{C}}(t, k)=\sup _{\alpha} \inf _{\beta} E\left[R\left(K_{T \wedge \tau^{\mathrm{C}}}^{t, k, \alpha, \beta}\right)\right] .
$$

The following proposition says that $\bar{V}^{\mathrm{C}}$ converges to $V$ as $C \rightarrow \infty$. Moreover, the proof reveals that the rate of convergence is exponential.

Proposition 3.1. Let $\mathrm{C} \in \mathbb{N}$. Then $\bar{V}^{\mathrm{C}}$ is the unique $C^{1}$ solution of

$$
\begin{cases}-\bar{V}_{t}^{\mathrm{C}}(t, k)=H\left(t, k, \bar{V}^{\mathrm{C}}\right), & \text { for } k=-\mathrm{C}+1, \ldots, \mathrm{C}-1, \\ \bar{V}^{\mathrm{C}}(t, \mathrm{C})=R(\mathrm{C}), \bar{V}^{\mathrm{C}}(t,-\mathrm{C})=R(-\mathrm{C}), & \text { for } t \in[0, T], \\ \bar{V}^{\mathrm{C}}(T, k)=R(k), & \text { for } k=-\mathrm{C}, \ldots, \mathrm{C} .\end{cases}
$$

Moreover, we have $\lim _{\mathrm{C} \rightarrow+\infty} \sup _{t \in[0, T]} \sup _{k \in[-\mathrm{C}, \mathrm{C}]}\left|\bar{V}^{\mathrm{C}}-V\right|=0$.

Before we prove the previous proposition, we introduce an auxiliary lemma. 
Lemma 3.2. There exists a constant $\mathrm{D}$, independent of $\mathrm{C}$, such that for $a \in A$ and $b \in B$

$$
\mathrm{P}\left(N_{T}^{\lambda_{1}(a, b)}-N_{t}^{\lambda_{1}(a, b)}>\frac{\mathrm{C}}{2}\right) \leq \mathrm{D} e^{-\mathrm{C} / 2}
$$

and

$$
\mathrm{P}\left(M_{T}^{\lambda_{2}(a, b)}-M_{t}^{\lambda_{2}(a, b)}>\frac{\mathrm{C}}{2}\right) \leq \mathrm{D} e^{-\mathrm{C} / 2} .
$$

Proof. Note that

$$
\begin{aligned}
\mathrm{P}\left(N_{T}^{\lambda_{1}(a, b)}-N_{t}^{\lambda_{1}(a, b)}>\frac{\mathrm{C}}{2}\right) & \leq e^{-\mathrm{C} / 2} E\left(e^{N_{T}^{\lambda_{1}(a, b)}-N_{t}^{\lambda_{1}(a, b)}}\right) \\
& \leq e^{-\mathrm{C} / 2} E\left(e^{N_{T}^{\lambda_{1}(a, b)}}\right)
\end{aligned}
$$

Note that $\lambda_{1}$ is bounded, say by $\bar{\lambda}$. Thus we get $E\left(e^{N_{T}^{\lambda_{1}(a, b)}}\right) \leq e^{-\bar{\lambda}(1-e)}$.

Proof of Proposition 3.1. Adapting the proof of Proposition 2.3 and Theorem 2.4, we obtain the first assertion.

To prove the second assertion, note that if $M>0$ is an upper bound for the reward function $R$, then

$$
\left|V(t, k)-\bar{V}^{\mathrm{C}}(t, k)\right| \leq M \sup _{\alpha, \beta} \mathrm{P}\left(\sup _{t \leq s \leq T}\left|K_{s}^{k, t, \alpha, \beta}\right| \geq \mathrm{C}\right) .
$$

Using the explicit form of $K$, we get

$$
\begin{aligned}
\mathrm{P}\left(\sup _{t \leq s \leq T}\left|K_{s}^{k, t, \alpha, \beta}\right| \geq \mathrm{C}\right) \leq & \mathrm{P}\left(N_{T}^{\lambda_{1}(\alpha, \beta)}-N_{t}^{\lambda_{1}(\alpha, \beta)} \geq \frac{\mathrm{C}}{2}\right) \\
& +\mathrm{P}\left(M_{T}^{\lambda_{2}(\alpha, \beta)}-M_{t}^{\lambda_{2}(\alpha, \beta)} \geq \frac{\mathrm{C}}{2}\right) .
\end{aligned}
$$

Lemma 3.2 leads to $\lim _{\mathrm{C} \rightarrow+\infty} \sup _{t \in[0, T]} \sup _{k \in[-\mathrm{C}, \mathrm{C}]}\left|\bar{V}^{\mathrm{C}}-V\right|=0$.

\subsubsection{Convergence of the time discretization}

The second step is to prove that the numerical approximation converges to the Isaacs solution on the compact set $[-\mathrm{C}, \mathrm{C}]$ as the time discretization goes to zero.

Theorem 3.3. We have

$$
\lim _{N \rightarrow+\infty} \sup _{k \in[-\mathrm{C}, \mathrm{C}]} \sup _{n \in[0, N]}\left|\bar{V}^{\mathrm{C}}\left(t_{n}, k\right)-\widetilde{V}^{\mathrm{C}}\left(t_{n}, k\right)\right|=0 .
$$


Proof. We adapt the standard proof of finite difference schemes for ODE (see e.g. [13]).

Stability: The numerical scheme is stable in the following sense: if $y$ and $\tilde{y}$ solve

$$
\begin{aligned}
& \tilde{y}\left(t_{n-1}, k\right)=\tilde{y}\left(t_{n}, k\right)+\Delta t H\left(t_{n}, k, \tilde{y}\right) \\
& y\left(t_{n-1}, k\right)=y\left(t_{n}, k\right)+\Delta t H\left(t_{n}, k, y\right)+\epsilon_{n}(k)
\end{aligned}
$$

with the boundary conditons $\tilde{y}(\cdot, \mathrm{C})=y(\cdot, \mathrm{C})=R(\mathrm{C})$ and $\tilde{y}(\cdot,-K)=y(\cdot,-K)=$ $R(-K)$, then

$$
\max _{n \in[0, N]} \max _{k \in[-\mathrm{C}, \mathrm{C}]}\left|\tilde{y}\left(t_{n}, k\right)-y\left(t_{n}, k\right)\right| \leq e^{L T}\left(\max _{k \in[-\mathrm{C}, \mathrm{C}]}\left|\tilde{y}\left(t_{N}, k\right)-y\left(t_{N}, k\right)\right|+\sum_{i=0}^{N} \max _{k \in[-\mathrm{C}, \mathrm{C}]}\left|\epsilon_{n}(k)\right|\right),
$$

where $L$ is the Lipschitz constant of $H$ (sometimes this property is referred as zerostablility, see [8]). Indeed, one has

$$
\begin{aligned}
\left|\tilde{y}\left(t_{n-1}, k\right)-y\left(t_{n-1}, k\right)\right| & =\left|\tilde{y}\left(t_{n}, k\right)-y\left(t_{n}, k\right)+\Delta t\left[H\left(t_{n}, k, \tilde{y}\right)-H\left(t_{n}, k, y\right)\right]-\epsilon_{n}(k)\right| \\
& \leq(1+\Delta t L) \max _{k \in[-K, K]}\left|\tilde{y}\left(t_{n}, k\right)-y\left(t_{n}, k\right)\right|+\max _{k \in[-\mathrm{C}, \mathrm{C}]}\left|\epsilon_{n}(k)\right| .
\end{aligned}
$$

It follows that

$$
\max _{k \in[-\mathrm{C}, \mathrm{C}]}\left|\tilde{y}\left(t_{n-1}, k\right)-y\left(t_{n-1}, k\right)\right| \leq(1+\Delta t L) \max _{k \in[-\mathrm{C}, \mathrm{C}]}\left|\tilde{y}\left(t_{n}, k\right)-y\left(t_{n}, k\right)\right|+\max _{k \in[-\mathrm{C}, \mathrm{C}]}\left|\epsilon_{n}(k)\right|
$$

Applying the discrete Gronwall Lemma to Equation (25), we obtain Equation (23).

Consistency: For every $k \in[-\mathrm{C}, \mathrm{C}]$ we define the consistency error

$$
e_{n}(k)=\bar{V}^{\mathrm{C}}\left(t_{n-1}, k\right)-\bar{V}^{\mathrm{C}}\left(t_{n}, k\right)-\Delta t H\left(t_{n}, k, \bar{V}^{\mathrm{C}}\right) .
$$

For all $k \in[-\mathrm{C}, \mathrm{C}]$, the function $\bar{V}^{\mathrm{C}}(\cdot, k)$ is in $\mathcal{C}^{1}$; therefore for each $\varepsilon>0$ there exists $N_{0}$ such that for each $N>N_{0}$

$$
\left|\bar{V}^{\mathrm{C}}(s, k)-\bar{V}^{\mathrm{C}}\left(t_{n}, k\right)\right| \leq \epsilon,
$$

for all $n, k \in[-\mathrm{C}, \mathrm{C}]$ and $s \in\left[t_{n-1}, t_{n}\right]$. Moreover, for fixed $n$ and $k$, there exists $s \in\left[t_{n-1}, t_{n}\right]$ such that

$$
\begin{aligned}
\left|e_{n}(k)\right| & =\Delta t\left|-\frac{\partial}{\partial t} \bar{V}^{\mathrm{C}}(s, k)-H\left(t_{n}, k, \bar{V}^{\mathrm{C}}\right)\right| \\
& \leq \Delta t\left|H\left(s, k, \bar{V}^{\mathrm{C}}\right)-H\left(t_{n}, k, \bar{V}^{\mathrm{C}}\right)\right| \\
& \leq \Delta t L \epsilon .
\end{aligned}
$$

The first inequality is obtained by using that $\bar{V}^{\mathrm{C}}$ is the solution of the Isaacs equation. The second inequality follows similar to the proof of Lemma 2.2 and Equation (26). 
Hence, taking the supremum in $k$, this entails

$$
\max _{k \in[-\mathrm{C}, \mathrm{C}]}\left|e_{n}(k)\right| \leq 2 \Delta t \max _{b \in B} \max _{a \in A}\left\{\lambda_{1}(a, b)+\lambda_{2}(a, b)\right\} \epsilon,
$$

and we obtain $\sum_{i=0}^{N-1} \max _{k \in[-\mathrm{C}, \mathrm{C}]}\left|e_{n}(k)\right| \leq T L \epsilon$. Since this holds true for each $\epsilon>0$ we get $\lim _{N \rightarrow+\infty} \sum_{i=0}^{N-1} \max _{k \in[-\mathrm{C}, \mathrm{C}]}\left|e_{n}(k)\right|=0$ and the numerical scheme is consistent with order 1.

Now the rest of the proof is standard. The stability and consistency imply the convergence of the scheme.

Remark 3.4. In the numerical experiments (see Section 4) we make the technical assumption that $\Delta t=\frac{T}{n}$ satisfies $1-\Delta t \max \left\{\lambda_{1}(a, b)+\lambda_{2}(a, b): a \in A, b \in B\right\}>0$. This guarantees that the approximating solution is positive for all $t$.

\subsection{Implicit finite difference scheme}

Similar to the explicit scheme we fix a localization parameter $C \in \mathbb{N}$, the number of time steps $N \in \mathbb{N}$ and set $t_{j}=j \Delta t$ with $\Delta t=\frac{T}{N}$, for $j \in\{0, \ldots, N\}$. Replacing the time derivative with a forward difference quotient we obtain

$$
\begin{cases}\widetilde{V}^{\mathrm{C}}\left(t_{n-1}, k\right)=\widetilde{V}^{\mathrm{C}}\left(t_{n}, k\right)+\Delta t H\left(t_{n-1}, k, \widetilde{V}^{\mathrm{C}}\right), & \text { for } 0 \leq n \leq N-1, k=-\mathrm{C}+1, \ldots, \mathrm{C}-1 \\ \widetilde{V}^{\mathrm{C}}\left(t_{n}, \mathrm{C}\right)=R(\mathrm{C}), \widetilde{V}^{\mathrm{C}}\left(t_{n},-K\right)=R(-K), & \text { for } 0 \leq n \leq N-1, \\ \widetilde{V}^{\mathrm{C}}(T, k)=R(k), & \text { for } k=-\mathrm{C}, \ldots, \mathrm{C} .\end{cases}
$$

Again one can prove convergence for the implicit scheme by showing stability and consistency. In contrast to the explicit scheme, we need the additional assumption $1-\Delta t L>0$ to ensure stability of the implicit scheme.

In the following we provide just the stability and consistency for the implicit scheme.

To prove convergence we refer to the proof of Theorem 3.3.

Similar to Inequality (24) we have

$$
\left|\tilde{y}\left(t_{n-1}, k\right)-y\left(t_{n-1}, k\right)\right| \leq\left|\tilde{y}\left(t_{n}, k\right)-y\left(t_{n}, k\right)+\Delta t\left[H\left(t_{n-1}, k, \tilde{y}\right)-H\left(t_{n-1}, k, y\right)\right]-\epsilon_{n}(k)\right|,
$$

where $y$ and $\tilde{y}$ are given by

$$
\begin{aligned}
& \tilde{y}\left(t_{n-1}, k\right)=\tilde{y}\left(t_{n}, k\right)+\Delta t H\left(t_{n-1}, k, \tilde{y}\right) \\
& y\left(t_{n-1}, k\right)=y\left(t_{n}, k\right)+\Delta t H\left(t_{n-1}, k, y\right)+\epsilon_{n}(k) .
\end{aligned}
$$

This entails

$$
\max _{k}\left|\tilde{y}\left(t_{n-1}, k\right)-y\left(t_{n-1}, k\right)\right| \leq \frac{1}{1-\Delta t L}\left(\max _{k}\left|\tilde{y}\left(t_{n}, k\right)-y\left(t_{n}, k\right)\right|+\max _{k}\left|\epsilon_{n}(k)\right|\right) .
$$

With the discrete Gronwall lemma we obtain

$$
\max _{k}\left|\tilde{y}\left(t_{n-1}, k\right)-y\left(t_{n-1}, k\right)\right| \leq \mathrm{e}^{T L} \max _{k}\left|\tilde{y}\left(t_{n}, k\right)-y\left(t_{n}, k\right)\right|+\frac{1}{L}\left(\mathrm{e}^{L T}-1\right) \sum_{i=0}^{N} \max _{k}\left|\epsilon_{n}(k)\right|
$$


and hence the stability is verified.

The consistency proof of the explicit scheme can be transferred, without further changes, to prove consistency of the implicit scheme since we are still working on the subintervals $\left[t_{n-1}, t_{n}\right]$. Hence we obtain convergence of the implicit scheme exactly the same way we do for the explicit scheme.

\subsection{Iterative scheme}

Recall that in Section 2 we prove existence and uniqueness of the Isaacs equation with the help of Banach's fixed point theorem. There is a natural approximation scheme connected to this approach. We approximate the fixed point operator $\Phi: \mathcal{X} \rightarrow \mathcal{X}$ with a sequence $\left(\Phi_{N}\right)$ of operators $\Phi_{N}: \mathcal{X} \rightarrow \mathcal{X}$, such that all $\Phi_{N}$ are contractions and the corresponding fixed points $w_{N}$, with $w_{N}=\Phi_{N}\left(w_{N}\right)$, converge to the fixed point $w$ of $\Phi$. It is well-known (cf. [16]) that the following two properties are sufficient for the converge of $w_{N}$ to $w$ :

(i) the contraction constants are uniformly bounded by a constant less than 1 ;

(ii) for each $x \in \mathcal{X}$ we have $\lim _{N \rightarrow \infty} \Phi_{N}(x)=\Phi(x)$.

Let $N \in \mathbb{N}$ and $\Delta t=\frac{T}{N}$. Set $t_{k}=k \cdot \Delta t$ for $k=1, \ldots, N$. We define the operator $\Phi_{N}$ by

$$
\Phi_{N}: \mathcal{X} \rightarrow \mathcal{X} ; \quad v \mapsto\left\{\begin{aligned}
\left((t, k) \mapsto R(k)-\sum_{i=j}^{N-1} H\left(t_{i}, k, v\right) \Delta t\right), & \text { if } t=t_{j}<t_{N} \\
\text { linear, } & \text { on }\left[t_{j}, t_{j+1}\right]
\end{aligned}\right.
$$

with boundary condition $(T, k) \mapsto R(k)$. One can prove that $\Phi^{N}$ is a contraction with the same contraction constant as $\Phi$. Hence the conditions $(i)$ and $(i i)$ are satisfied and we obtain convergence $w_{N} \rightarrow w$ as $N \rightarrow \infty$.

In order to study the rate of convergence we separate two types of approximation errors. First we get an error by using $\Phi_{N}$ instead of $\Phi$ (which solely depends on $N$ ), and an error made by the approximation of $w_{N}$ by iterating $\Phi_{N}$ over and over again (which solely depend on the number of iterations). Indeed, let $v_{m}^{N}$ be the approximation for given $N \in \mathbb{N}$ after $m$ iterations and $v_{\infty}^{N}=v^{N}$ the exact fixed point of $\Phi_{N}$, then

$$
\left\|v_{m}^{N}-w\right\|_{\infty} \leq\left\|v_{m}^{N}-v_{\infty}^{N}\right\|_{\infty}+\left\|v_{\infty}^{N}-w\right\|_{\infty} .
$$

Thanks to Banach's fixed point theorem the first term converges exponentially fast. Moreover, a straightforward estimation entails that the second term converges with rate $1 / N$, which gives in the end a rate of convergence of $\mathcal{O}(1 / N)$.

\section{Numerical experiments}

In this last section we present results from various numerical examples and experiments. Throughout we choose $A=B=[0,1]$ and assume that the reward function $R$ is given 
by (5). For approximating the Isaacs Equation (8) and the optimal strategies $\left(\alpha^{*}, \beta^{*}\right)$ we use the explicit finite difference scheme (see Subsection 3.1). The explicit scheme does not require any assumption on the time step to ensure the stability (contrary to the implicit scheme). For all experiments we choose $T=1, N=90$ and $C=10$. Moreover, we discretize the strategy spaces $A=B=[0,1]$ into the subset $\{m \cdot 0.01 \mid m \in$ $\{0,1, \ldots, 100\}\}$.

In some examples and experiments we assume that the intensity functions $\lambda_{i}$ are differentiable and additively decomposable into $\lambda_{1}(a, b)=f_{1}(a)+g_{1}(b)$ and $\lambda_{2}=g_{2}(a)+$ $f_{2}(b)$, where $f_{i}$ is concave and $g_{i}$ convex for $i \in\{1,2\}$. In this case the Nash equilibrium can be determined by solving the equations

$$
\begin{aligned}
& \frac{\partial G}{\partial a}=f_{1}^{\prime}(a) \Delta(k+1, t)-g_{2}^{\prime}(a) \Delta(k, t)=0, \\
& \frac{\partial G}{\partial b}=g_{1}^{\prime}(b) \Delta(k+1, t)-f_{2}^{\prime}(b) \Delta(k, t)=0
\end{aligned}
$$

where $\Delta(k, t)=V(k, t)-V(k-1, t)$. Notice that $\alpha^{*}$ depends on $\beta^{*}$ implicitly, because $\Delta(k, t)$ depends on both strategies.

\subsection{Do or die}

To illustrate the impact of the score on the optimal strategies we consider two examples.

The first example is additive with $\lambda_{1}(a, b)=\lambda_{2}(a, b)=f(a)+g(b)$, where

$$
f(x)=1-(1-x)^{2}(1-\sqrt{x}) \text { and } g(x)=1-f(1-x), \quad x \in[0,1] .
$$

Notice that $f$ is concave and $g$ is convex. Moreover, $f^{\prime}(0)=g^{\prime}(1)=\infty$ and $f^{\prime}(1)=$ $g^{\prime}(0)=0$. The own team does not choose the strategy 0 , because in 0 the marginal increase of the opponent's goal intensity is zero, whereas the marginal increase of the own intensity is infinite. Similarly, the own team does not choose the strategy 1 , because in 1 the marginal increase of the opponent's intensity is infinite, whereas the marginal increase of the own intensity is zero.

In this example both teams are equally strong. Therefore, whenever the game is tied, both teams choose the same action and score a goal with the same intensity. The strategies change significantly after a goal. Figure 1 shows the graph of $\alpha_{t}^{*}$ and $\beta_{t}^{*}$ as a function of time, conditional to the own team being behind by one goal, i.e. $K_{t}=-1$. The figure reveals that it is optimal for the winning team to switch to a more defensive strategy and for the losing team to a more offensive one.

The effect of the score on the strategies is already significant at the beginning of a game. The effect becomes the larger, the closer the end of the game. If there is no tie, then towards the end of the game the winning team starts playing extremely defensively and the losing team extremely offensively. In Figure 1, $\alpha_{t}^{*}$ converges to 1 , the most offensive action, and $\beta_{t}^{*}$ converges to 0 , the most defensive action, as time approaches the end of the game. Any spectator seeing both teams choosing extreme actions towards the end may have the impression of a last minute panic among the players. 
We now turn to a second example, with multiplicatively decomposable intensity functions. More precisely, we consider $\lambda_{1}(a, b)=\lambda_{2}(b, a)=(1+f(a)) \cdot(1+g(b))$, where $f$ and $g$ are again defined as in (33). As in the first example, both teams are equally strong and a change in the score has a strong impact on the strategies. Figure 2 depicts the graph of $\alpha^{*}$ and $\beta^{*}$, conditional to $K_{t}=-1$.

Both examples reveal that the winning team strives to reduce the scoring intensities whereas the loosing team aims at increasing them. Since both effects cancel out, we expect to observe a smaller impact of the score on the scoring intensities than on the actions. Indeed, as shown in the next subsection, there are situations where the effects cancel out fully and the scoring intensities are the same for any score and at any time.

The aggregate scoring intensity, i.e. the intensity of any goal scored by the own team or the opponent, is given by $\lambda_{1}+\lambda_{2}$. The part of the aggregate intensity allotted to the own team and the opponent is given by the ratios $\frac{\lambda_{1}}{\lambda_{1}+\lambda_{2}}$ and $\frac{\lambda_{2}}{\lambda_{1}+\lambda_{2}}$, respectively.

The losing team aims at increasing the scoring intensities. To this end it is willing to concede a part of the scoring ratio to the opponent. Similarly, the winning team aims at decreasing the scoring intensities and, for this purpose, is willing to concede a part of the scoring ratio. Whether in an equilibrium the winning team or the losing team concedes more, depends on which team has a stronger impact on the scoring intensities.

To illustrate this, consider a situation where only the own team can change its strategy, while the opponent always has to stick to the same action. In others words, the intensities $\lambda_{1}$ and $\lambda_{2}$ depend on $a$ but not on $b$. As soon as the own team falls behind, it switches to a more offensive strategy. By doing so it increases its own scoring intensity by a smaller factor than the opponent's intensity. Thus, the ratio $\frac{\lambda_{1}}{\lambda_{1}+\lambda_{2}}$ decreases. Conversely, as soon as the own team takes the lead, it starts playing more defensively. It thus reduces the own scoring intensity by a larger factor than the opponent's intensity. Again, the ratio $\frac{\lambda_{1}}{\lambda_{1}+\lambda_{2}}$ decreases. This exemplifies that in any case a team is willing to accept a relative increase of the opponent's scoring intensity in order to maximize its expected gain. The willingness remains also in a game with interaction, even though it may not be observed in the actual equilibrium.

\subsection{When goals don't reveal the strategies}

Suppose that the intensity functions of both teams are given by $\lambda_{1}(a, b)=\lambda_{2}(b, a)=$ $f(a)+g(b)$, where the functions $f$ and $g$ are strictly concave and convex, respectively, and satisfy $g(x)=1-f(1-x), \forall x \in[0,1]$. Notice that in this case both teams are equally strong and we have $g^{\prime}(a)=f^{\prime}(1-a)$ for all $a \in[0,1]$. The following result shows that in this case the scoring intensities do neither depend on time nor on the score.

Proposition 4.1. The unique Nash equilibrium $\left(\alpha^{*}, \beta^{*}\right)$ satisfies $\alpha^{*}(t, k)=1-\beta^{*}(t, k)$ and $\lambda_{i}\left(\alpha^{*}(t, k), \beta^{*}(t, k)\right)$ is constant equal to one for all $i=1,2$ and $(t, k) \in[0, T] \times \mathbb{Z}$. 


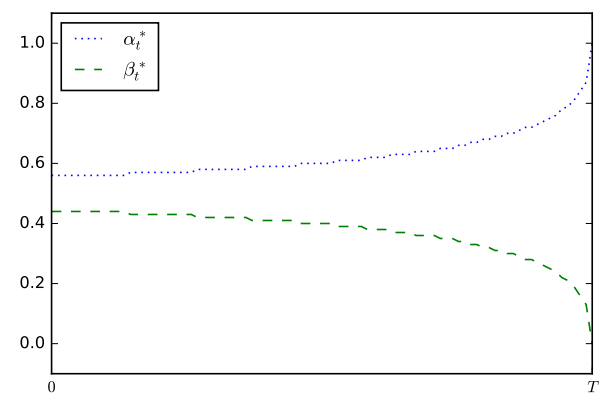

Figure 1: graph of $\alpha^{*}$ and $\beta^{*}$ conditional to $K_{t}=-1$ in the additive example.

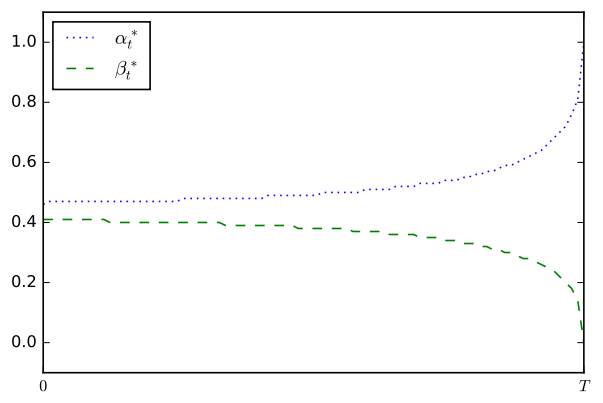

Figure 2: graph of $\alpha^{*}$ and $\beta^{*}$ conditional to $K_{t}=-1$ in the multiplicative example.

Proof. As the intensities are additive and symmetric, the first order condition is

$$
\begin{aligned}
& \frac{\partial G}{\partial a}=f^{\prime}(a) \Delta(k+1, t)-f^{\prime}(1-a) \Delta(k, t)=0, \\
& \frac{\partial G}{\partial b}=f^{\prime}(1-b) \Delta(k+1, t)-f^{\prime}(b) \Delta(k, t)=0 .
\end{aligned}
$$

This implies that $\beta^{*}(t, k)=1-\alpha^{*}(t, k)$. Indeed, we first remark that $\frac{\partial G}{\partial a}$ is decreasing and $\frac{\partial G}{\partial b}$ is increasing. We can distinguish the following cases.

1. There is a unique solution $\alpha^{*}(t, k)$ to equation (34) in $] 0,1\left[\right.$. In this case $\beta^{*}(t, k)=$ $1-\alpha^{*}(t, k)$ is a solution to $(35)$.

2. For all we have $a \in] 0,1\left[, \frac{\partial G}{\partial a}>0\right.$. In this case $\alpha^{*}(t, k)=1$ and $\beta^{*}(t, k)=0$.

3. For all we have $a \in] 0,1\left[, \frac{\partial G}{\partial a}<0\right.$. In this case $\alpha^{*}(t, k)=0$ and $\beta^{*}(t, k)=1$.

We further obtain

$$
\lambda_{1}\left(\alpha^{*}(t, k), \beta^{*}(t, k)\right)=f\left(\alpha^{*}(t, k)\right)+g\left(\beta^{*}(t, k)\right)=f\left(\alpha^{*}(t, k)\right)+1-f\left(\alpha^{*}(t, k)\right)=1 .
$$

The same result follows for $\lambda_{2}\left(\alpha^{*}(t, k), \beta^{*}(t, k)\right)$.

Notice that the additive example of Subsection 4.1 satisfies the assumptions of Proposition 4.1. This example reveals that the score can have a large impact on the strategies, but not impact at all on the scoring intensities (cf. Figure 1).

\subsection{Are weaker teams more likely to win in football than in basketball?}

It can happen that a team wins the game although it is weaker than the other one. Our numerical experiments show that the likelihood for the weaker team to win depends on 


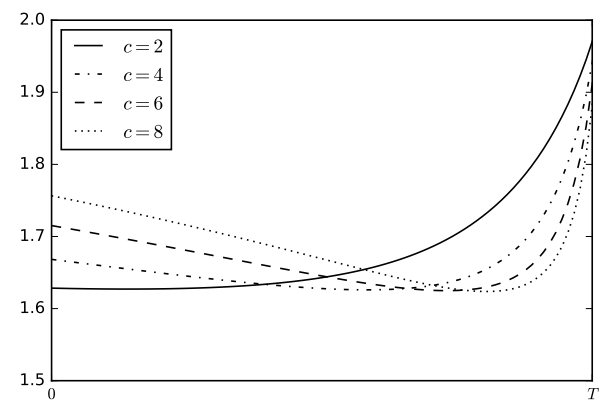

Figure 3: Expected reward of the better team if it is leading by one goal.

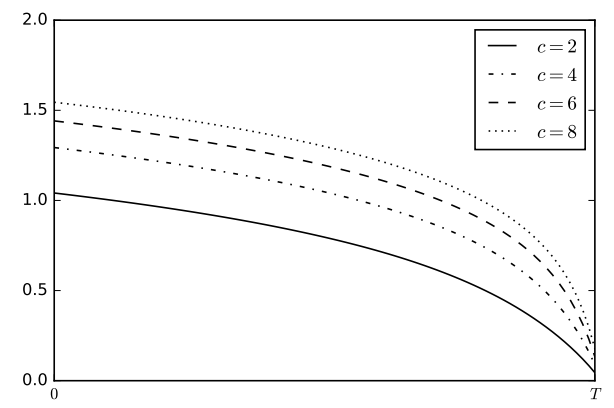

Figure 4: Expected reward of the better team if it is behind by one goal.

the average number of goals that are scored in typical games of the sport considered. To illustrate the dependence, consider the situation where the own team scores, in average, 1,5 times more often than the opposing team. More precisely, let $f$ and $g$ be defined as in (33), and set $\lambda_{1}(a, b)=c(f(a)+g(b))$ and $\lambda_{2}(a, b)=\frac{2}{3} \lambda_{1}(b, a)$, where $c \in\{2,4,6,8\}$.

Figure 3 shows the expected reward of the own team, conditional to being ahead by one goal, i.e. $K_{t}=1$. A higher scoring intensity entails that both teams score more often and hence the expected scoring lead at $T$ of the better team becomes larger. Therefore, at the beginning of the game the expected reward of the better team increases with the overall scoring intensity. In other words, weaker teams are more likely to win in soccer than in basketball. Shortly before the end of the game, however, a high intensity can be an advantage for the weaker team if it is behind. A higher overall intensity implies a larger likelihood for the weaker team to equalize before the end of the game.

Figure 4 shows the expected reward of the own team, conditional to being behind by one goal, i.e. $K_{t}=-1$. In this case, a higher overall scoring intensity is an advantage for the better team at any time of the game.

\section{A. Appendix}

In this appendix we explain how to choose a filtered probability space $\left(\Omega, \mathcal{F},\left(\mathcal{F}_{t}\right), \mathrm{P}\right)$ such that for every $\alpha \in \mathcal{A}$ and $\beta \in \mathcal{B}$ we can define point processes $N$ and $M$ with intensity $\lambda_{1}\left(\alpha\left(t, K_{t}^{\alpha, \beta}\right), \beta\left(t, K_{t}^{\alpha, \beta}\right)\right)$ and $\lambda_{2}\left(\alpha\left(t, K_{t}^{\alpha, \beta}\right), \beta\left(t, K_{s}^{\alpha, \beta}\right)\right)$ respectively.

Consider a probability space $(\Omega, \mathcal{F}, \mathrm{P})$ endowed with a Poisson random measure $\mu$. Recall that this means that for all $B \in \mathcal{B}(\mathbb{R})$ with $\lambda(B)<\infty$ the process $t \mapsto \mu([0, t] \times$ $B)$ is a Poisson process with parameter $\lambda(B)$, where $\lambda$ denotes the Lebesgue measure. Moreover, the Poisson processes corresponding to two disjoint Borel sets are independent. Now we set $\mathcal{F}_{t}:=\sigma\left(X_{s}^{B}: s \leq t, B \in \mathcal{B}(\mathbb{R})\right)$, where $X_{s}^{B}:=\mu([0, s] \times B)$. This gives us a suitable filtration without the need to know $K^{\alpha, \beta}$. 
We define $K^{\alpha, \beta}$ as the solution of the stochastic integral equation

$$
K_{t}^{\alpha, \beta}=\int_{0}^{t} \int_{\mathbb{R}}\left[\mathbb{1}_{\left[0, \lambda_{1}\left(\alpha\left(t, K_{s}^{\alpha, \beta}\right), \beta\left(t, K_{s}^{\alpha, \beta}\right)\right)\right]}(u)-\mathbb{1}_{\left[-\lambda_{2}\left(\alpha\left(t, K_{s}^{\alpha, \beta}\right), \beta\left(t, K_{s}^{\alpha, \beta}\right)\right), 0\right]}(u)\right] \mu(\mathrm{d} s, \mathrm{~d} u)
$$

and

$$
\begin{aligned}
N_{t}^{\lambda_{1}(\alpha, \beta)} & :=\int_{0}^{t} \int_{\mathbb{R}} \mathbb{1}_{\left[0, \lambda_{1}\left(\alpha\left(s, K_{s}^{\alpha, \beta}\right), \beta\left(s, K_{s}^{\alpha, \beta}\right)\right)\right]}(u) \mu(\mathrm{d} s, \mathrm{~d} u), \\
M_{t}^{\lambda_{2}(\alpha, \beta)} & :=\int_{0}^{t} \int_{\mathbb{R}} \mathbb{1}_{\left[-\lambda_{2}\left(\alpha\left(s, K_{s}^{\alpha, \beta}\right), \beta\left(s, K_{s}^{\alpha, \beta}\right)\right), 0\right]}(u) \mu(\mathrm{d} s, \mathrm{~d} u) .
\end{aligned}
$$

Standard arguments (see e.g. $§$ 1.c, Chapter II, [9]) imply that $N^{\lambda_{1}(\alpha, \beta)}$ and $M^{\lambda_{2}(\alpha, \beta)}$ are point processes with intensity $\lambda_{1}(\alpha, \beta)$ and $\lambda_{2}(\alpha, \beta)$ respectively.

The definitions (37) and (38) entail that the increments of $N$ and $M$ between two consecutive jumps are conditionally independent and Poisson distributed. More precisely, for $0 \leq s<t, \rho:=\inf \left\{u \geq s: K_{u} \neq K_{s}\right\}$ and $i, j \in\{0,1\}$ with $i+j \leq 1$, we have

$$
\begin{aligned}
& \mathrm{P}\left(N_{t \wedge \rho}^{\lambda_{1}}-N_{s}^{\lambda_{1}}=i, M_{t \wedge \rho}^{\lambda_{2}}-M_{s}^{\lambda_{2}}=j \mid \mathcal{F}_{s}\right) \\
& =\mathrm{P}\left(N_{t \wedge \rho}^{\lambda_{1}}-N_{s}^{\lambda_{1}}=i \mid \mathcal{F}_{s}\right) \mathrm{P}\left(M_{t \wedge \rho}^{\lambda_{2}}-M_{s}^{\lambda_{2}}=j \mid \mathcal{F}_{s}\right) \\
& =\mathrm{e}^{-\int_{s}^{t \wedge \rho}\left(\lambda_{1}(u)+\lambda_{2}(u)\right) \mathrm{d} u}\left(\int_{s}^{t \wedge \rho} \lambda_{1}(u) \mathrm{d} u\right)^{i}\left(\int_{s}^{t \wedge \rho} \lambda_{2}(u) \mathrm{d} u\right)^{j} .
\end{aligned}
$$

\section{References}

[1] S. Ankirchner, C. Blanchet-Scalliet, and M. Jeanblanc. Controlling the occupation time of an exponential martingale. Applied Mathematics \& Optimization, pages $1-14,2015$.

[2] J.-P. Aubin. Mathematical methods of game and economic theory, volume 7 of Studies in Mathematics and its Applications. North-Holland Publishing Co., Amsterdam-New York, 1979.

[3] M. Bardi, T. E. S. Raghavan, and T. Parthasarathy, editors. Stochastic and differential games, volume 4 of Annals of the International Society of Dynamic Games. Birkhäuser Boston, Inc., Boston, MA, 1999. Theory and numerical methods.

[4] D. Beaudoin and T. B. Swartz. Strategies for pulling the goalie in hockey. The American Statistician, 2012.

[5] P. Brémaud. Bang-bang controls of point processes. Advances in Appl. Probability, 8(2):385-394, 1976. 
[6] M. Falcone. Numerical methods for differential games based on partial differential equations. International Game Theory Review, 8(02):231-272, 2006.

[7] M. Falcone and R. Ferretti. Semi-Lagrangian Approximation Schemes for Linear and Hamilton - Jacobi Equations. Society for Industrial and Applied Mathematics, Philadelphia, PA, 2013.

[8] E. Hairer, S. Nørsett, and G. Wanner. Solving Ordinary Differential Equations I, volume 8 of Springer Series in Computational Mathematics. Springer-Verlag Berlin Heidelberg, 1993.

[9] J. Jacod and A. N. Shiryaev. Limit theorems for stochastic processes, volume 288 of Grundlehren der Mathematischen Wissenschaften [Fundamental Principles of Mathematical Sciences]. Springer-Verlag, Berlin, second edition, 2003.

[10] P. Jaillet, D. Lamberton, and B. Lapeyre. Variational inequalities and the pricing of American options. Acta Appl. Math., 21(3):263-289, 1990.

[11] J. McNamara. Optimal control of the diffusion coefficient of a simple diffusion process. Mathematics of Operations Research, 8(3):373-380, 1983.

[12] D. G. Morrison and R. D. Wheat. Misapplications reviews: Pulling the goalie revisited. Interfaces, 16(6):28-34, 1986.

[13] L. F. Shampine. Numerical solution of ordinary differential equations. Chapman \& Hall, New York, 1994.

[14] M. Sion. On general minimax theorems. Pacific J. Math., 8:171-176, 1958.

[15] A. Washburn. Still more on pulling the goalie. Interfaces, 21(2):59-64, 1991.

[16] E. Zeidler. Nonlinear functional analysis and its applications. I. Springer-Verlag, New York, 1986. Fixed-point theorems, Translated from the German by Peter R. Wadsack.

[17] X. L. Zhang. Numerical analysis of American option pricing in a jump-diffusion model. Math. Oper. Res., 22(3):668-690, 1997. 